\section{Se doter de principes directeurs pour les prescripteurs électroniques de médicaments? L'exemple d'un centre hospitalier universitaire}

Dans le Rapport 2011-2012 sur les pharmacies hospitalières canadiennes, Macgregor et Hall notent que "l'intégration des systèmes et l'accès en temps réel à l'information au point de service posent toujours des défis aux hôpitaux du Canada, même si les publications indiquent que ces systèmes peuvent réduire les erreurs, améliorer les décisions et avoir un effet positif sur le soin des patients et les résultats cliniques $»^{1}$. Georgiou et coll. ont mené une revue systématique sur les systèmes de "saisie électronique des ordonnances par les médecins " (SEOM) aux urgences $^{2}$. Du nombre limité d'articles de qualité retenus $(n=22)$, on retient que les SEOM augmentent le temps passé à l'ordinateur par les médecins $(11 \%)$ et les infirmières (16\%) sans réduire le temps passé au chevet des patients, qu'ils sont associés à des réductions d'erreurs médicamenteuses (de l'ordre de 17 à 201 erreurs/100 ordonnances), à des réductions du nombre d'effets indésirables potentiels (0,9 effets/100 ordonnances) et à des diminutions du nombre de prescriptions liées à un dosage trop élevé. D'autres auteurs nuancent ces avantages et questionnent les effets cliniques réels des $\mathrm{SEOM}^{3-6}$.

Pedersen et coll. rapportent qu'en 2010, aux États-Unis, 16 \% des hôpitaux (29 \% des hôpitaux de 300-399 lits, 30 \% des hôpitaux de 400-599 lits et 57 \% des hôpitaux de plus de 600 lits) avaient déjà implanté un $\mathrm{SEOM}^{7}$. Notons que moins de $4 \%$ de ces implantations ne comportent aucune interface avec le système d'information de la pharmacie. Macgregor et Hall note qu'au Canada, seulement $8 \%$ des répondants (13/160 établissements) ont déjà implanté un SEOM, et près des trois quarts ont une interface avec le système d'information de la pharmacie'.

Plusieurs facteurs contribuent à la lenteur de l'implantation de SEOM tant aux États-Unis qu'au Canada, notamment le fait que de nombreux secteurs s'étaient progressivement informatisés durant les dernières décennies (p. ex. admission, pharmacie, laboratoires, imagerie) avant d'envisager l'acquisition d'un SEOM ainsi que le fait que les SEOM entraînent des coûts d'acquisition très élevés et qu'ils sont complexes à implanter dans une organisation de santé. Plusieurs auteurs reconnaissent l'importance d'une interface bidirectionnelle entre le SEOM et le système d'information de pharmacie $e^{8-10}$. Au moins trois documents pivots balisent actuellement le déploiement de SEOM au Canada, soit la norme relative aux dossiers médicaux électroniques d'Inforoute Canada, les normes relatives aux messages de type HL7, la norme sur les systèmes d'information en pharmacie de l'Association nationale des organismes de réglementation en pharmacie ${ }^{11-13}$. L'Ordre des pharmaciens du Québec a mis en place un comité de veille technologique qui réfléchit notamment à la mise en place de lignes directrices portant sur les systèmes d'information ${ }^{14}$.

Dans la perspective d'assurer des acquisitions logicielles sécuritaires et compatibles avec la réglementation et les systèmes en place intégrant les avancées technologiques et toutes les étapes du circuit du médicament, le comité de pharmacologie du Centre hospitalier universitaire (CHU) Sainte-Justine s'est doté en avril 2013 de principes directeurs pour toute acquisition et intégration de solutions logicielles de SEOM. Ces principes découlent d'une revue documentaire, de visites de sites ayant implanté des SEOM ailleurs au Canada et d'analyses faisant suite à l'implantation d'autres projets au sein de l'établissement. Les principes ont été discutés lors de réunions du département de pharmacie avant d'être proposés aux membres du comité de pharmacologie. Ils ont aussi été présentés au comité exécutif du Conseil des médecins, dentistes et pharmaciens. Ils ont en outre été diffusés par courriels à tous les médecins, pharmaciens et cadres de l'établissement en guise de suivi de la réunion du comité de pharmacologie. La diffusion de ces principes directeurs peut contribuer à soutenir les démarches de réflexion et de prises de décisions au sein des établissements canadiens et faciliter l'adoption de bonnes pratiques informatiques pour éviter que des SEOM ne soient acquis et implantés sans interface avec le système d'information de pharmacie. Si l'acquisition de solutions incorporant le SEOM et les systèmes d'information de laboratoire, de pharmacie et d'autres disciplines permet cette intégration souhaitable, d'autres options existent, mais il faut que les systèmes soient interfacés de façon appropriée. Dans tous les cas, un pharmacien du département de pharmacie devrait être désigné pour veiller à l'introduction et à l'utilisation appropriées des technologies de l'information ${ }^{15}$. Le boîte 1 présente les principes directeurs adoptés et désormais en vigueur au sein de l'organisation.

\section{Références}

1. Macgregor P, Hall K. Chapitre E: Technologie. Dans : Babich M, Bornstein C, Bussières JF, Doucette D, Hall K, Lefebvre P, et coll., comité de rédaction. Rapport annuel 2011 -2012 sur les pharmacies hospitalières canadiennes. Eli Lilly; 2012. p. 44-54. Publié au http://www.lillyhospitalsurvey.ca/hpc2/ content/2012_report/chapter_eF\%20.pdf. Consulté le 5 août 2013.

2. Georgiou A, Prgomet M, Paoloni R, Creswick N, Hordern A, Walter S, et al. The effect of computerized provider order entry systems on clinical care and work processes in emergency departments: a systematic review of the quantitative literature. Ann Emerg Med. 2013;61(6):644-53.e16.

3. Radley DC, Wasserman MR, Olsho LE, Shoemaker SJ, Spranca MD, Bradshaw B. Reduction in medication errors in hospitals due to adoption of computerized provider order entry systems. J Am Med Inform Assoc. 2013; 20(3):470-6.

4. Abramson EL, Kaushal R. Computerized provider order entry and patient safety. Pediatr Clin North Am. 2012;59(6):1247-55.

5. Manias E, Williams A, Liew D. Interventions to reduce medication errors in adult intensive care: a systematic review. Br J Clin Pharmacol. 2012;74(3): 411-23.

6. Weir CR, Staggers N, Laukert T. Reviewing the impact of computerized provider order entry on clinical outcomes: the quality of systematic reviews. Int J Med Inform. 2012;81(4):219-31. 
Boîte 1. Principes directeurs pour la planification et l'acquisition de saisie électronique des ordonnances par les médecins (SEOM) en établissement de santé au Centre hospitalier universitaire Sainte-Justine (CHUSJ)

Tout cahier des charges rédigé pour un appel d'offres de nouveaux prescripteurs électroniques de médicaments, tout contrat de services pour un prescripteur électronique de médicaments ou toute démarche d'évaluation pour une acquisition éventuelle d'un prescripteur au CHUSJ doit inclure l'ensemble des principes directeurs décrits ci-après. L'octroi d'un contrat doit aller au meilleur soumissionnaire, tenir compte de ce qui est réellement disponible et de ce à quoi s'engagent les soumissionnaires et être accompagné d'un échéancier.

Il doit y avoir idéalement un seul prescripteur électronique de médicaments (ou ils doivent être en nombre très limité) au sein d'un même établissement, ce qui favorise la réduction des coûts, l'allégement des ressources requises pour le maintien de ces systèmes informatiques, la mise à jour des bases de données ainsi que la formation requise des utilisateurs. Le prescripteur uniquediminue également les risques d'incidents-accidents inhérents à la multiplicité des systèmes et à la complexification des processus de prescription et d'utilisation de médicaments dans le circuit.

Tout projet de déploiement d'un SEOM doit respecter les exigences suivantes :

- Tout SEOM doit comporter une interface bidirectionnelle conforme au dossier pharmacologique informatisé (DP); une interface conforme requiert la capacité de transmettre des ordonnances conformes aux besoins du DP et la capacité de recevoir des commentaires du DP; en l'absence $d^{\prime}$ 'interface bidirectionnelle, une feuille d'administration des médicaments (FADM) papier issue du DP doit être utilisée; une FADM conforme est essentielle au respect de la réglementation fédérale et des responsabilités de l'établissement en matière de surveillance des substances contrôlées (c.-à-d. stupéfiants, drogues contrôlées, substances ciblées et benzodiazépines).

Tout SEOM doit comporter des tables conformes aux spécifications du DP afin d'utiliser le même dictionnaire de données, sans troncature des renseignements.

Tout SEOM doit permettre la rédaction d'ordonnances électroniques entièrement conformes à la règle d'émission des ordonnances adoptées par le Comité de pharmacologie, le Conseil des médecins, dentistes et pharmaciens et le Conseil d'administration.

Tout SEOM doit afficher de façon obligatoire les commentaires de validation pharmaceutique provenant du DP avant qu'un médicament ne puisse être administré par le personnel soignant ; dans la perspective où le SEOM permet l'enregistrement des doses sous forme de FADM électronique, I'ensemble des règles inhérentes à l'enregistrement des doses, y compris le recours aux codes-barres en vigueur, doit être respecté.

Tout SEOM doit comporter des outils permettant de vérifier en temps réel l'intégrité référentielle des opérations, y compris les transactions orphelines détectées lors de transactions faites au moyen de l'interface bidirectionnelle.

- Tout SEOM doit permettre de gérer des protocoles de médicaments (c.-à-d. l'équivalent de feuilles d'ordonnances prérédigées avec ordonnances liées) et de permettre la saisie, le contrôle de qualité et le déploiement de nouveaux protocoles de médicaments; le processus distinct préalable à cette saisie de protocoles de médicaments doit permettre de renseigner les auteurs et les réviseurs du contenu scientifique, et cette documentation papier doit uniquement servir à la vérification de l'intégrité des ordonnances ou être utilisée en cas d'incidentsaccidents ou de litiges juridiques; le chef du département de pharmacie est responsable de l'archivage de ces protocoles et de la conformité du processus d'approbation; ce processus préalable est en vigueur au CHUSJ depuis plus d'une décennie.

- Tout SEOM doit permettre l'utilisation et la lecture de codes-barres conformes sur des médicaments ou des documents (p. ex. cartes d'employé, documents papier, formulaires) selon les standards en vigueur au CHUSJ ( $p$. ex. GS1) pour l'enregistrement des doses de médicaments administrées par le personnel soignant au moyen du lecteur code-barres.

- Toute implantation de SEOM doit comporter des modes de fonctionnement de secours en cas d'urgence (p. ex. plan d'urgence comportant différents scénarios, tels que panne de réseau, panne de serveur, panne de postes locaux, panne logicielle).

- Tout SEOM doit permettre de réaliser la démarche du bilan comparatif des médicaments à l'admission et au transfert de patients (p. ex. transfert d'unités de soins, congé de l'établissement); le SEOM doit pouvoir consulter électroniquement le bilan comparatif dans le cadre habituel de prescription de médicaments durant le séjour et au congé.

- Tout SEOM doit permettre au personnel soignant de déterminer le statut de validation pharmaceutique des ordonnances en temps réel afin d'encadrer l'administration sécuritaire des médicaments.

- Tout SEOM doit permettre aux personnes requises de gérer efficacement les ordonnances verbales et les contresignatures a posteriori dans un délai prédéterminé.

- Tout projet SEOM doit comporter la mise en place d'une équipe de soutien technique et clinique ( $p$. ex. mise à jour des bases de données en temps réel) y compris des modalités de continuité 24 heures sur 24; ce personnel doit être identifié et avoir assez de temps pour assurer l'intégrité et la qualité des données; en outre, toute mise à jour des bases de données concernant le médicament doit se faire selon des politiques et procédures approuvées conjointement par le chef du département de pharmacie et les responsables des différents progiciels. 
7. Pedersen CA, Schneider PJ, Scheckelhoff DJ. ASHP national survey of pharmacy practice in hospital settings: prescribing and transcribing-2010. Am J Health Syst Pharm. 2011;68(8):669-88.

8. Chaffee BW, Bonasso J. Strategies for pharmacy integration and pharmacy information system interfaces, Part 1: History and pharmacy integration options. Am J Health Syst Pharm. 2004;61(5):502-6.

9. Holdford DA, Huffines SK, Rosenbloom ST. Chapitre 9 : Electronic data management: electronic health record systems and computerized provider order entry systems. Dans : Holdford DA, Brown TR, redacteurs. Introduction to hospital and health-system pharmacy practice. Bethesda (MD) : American Society of Health-System Pharmacists; 2010. p. 159-78.

10. ASHP guidelines on pharmacy planning for implementation of computerized provider-order entry systems in hospitals and health systems. Bethesda (MD) : American Society of Health-System Pharmacists; 2011. Publié au www.ashp.org/DocLibrary/BestPractices/AutoITGdlCPOE.aspx. Consulté le 5 août 2013.

11. Dossiers médicaux électroniques (DME). Montréal (QC) : Inforoute Santé du Canada; 2012. Publié au https://www.infoway-inforoute.ca/index.php/fr/ programmes-services/services-de-certification/ce-quinforoute-certifie/dossiersmedicaux-electroniques-dme. Consulté le 5 août 2013.

12. EMR specifications. Dans : HL7 v3 pan-Canadian messaging standards. Implementation guide volume 8-pharmacy. Version R02.04.00. Inforoute Santé du Canada; 16 mars 2009.

13. Pharmacy practice management systems: requirements to support NAPRA standards of practice. Ottawa $(\mathrm{ON})$ : Association nationale des organismes de réglementation de la pharmacie; 4 novembre 2012.
14. Rapport annuel 2012-2013. Montréal (QC) : Ordre des pharmaciens du Québec, Comité de veille sur les nouvelles pratiques liés aux développements technologiques. Publié au : www.opq.org/cms/Media/1585_38_ fr-CA_0_Rapport_annuel_2012_2013_OPQ.pdf. Consulté juin 2013.

15. ASHP statement on the pharmacist's role in informatics. Bethesda (MD) : American Society of Health-System Pharmacists; 2006. Publié au : www.ashp.org/DocLibrary/BestPractices/AutoITStInformatics.aspx. Consulté le 5 août 2013 .

Denis Lebel, B. Pharm., M.Sc., FCSHP

Adjoint

Jean-François Bussières, B. Pharm., M.Sc., FCSHP

Chef

Département de pharmacie et Unité de recherche en pratique

pharmaceutique

Centre hospitalier universitaire Sainte-Justine

Montréal, Québec

Jean-François Bussières est aussi professeur titulaire de clinique, Faculté de pharmacie, Université de Montréal, Montréal, Québec.

Intérêts concurrents : aucun déclaré.

\section{CJHP Subscriptions 20I4 / Abonnements au JCPH 2014}

CSHP has introduced both Print and Print + Online pricing models for CJHP subscriptions. Print + Online CJHP is included as a benefit of CSHP membership. All prices are in Canadian funds.

La SCPH a établi une grille tarifaire pour l'abonnement à la copie imprimée du JCPH seulement et pour l'abonnement à la fois aux copies imprimée et électronique du journal. L'abonnement combiné est inclus dans les droits d'adhésion à la SCPH. Tous les prix sont en dollars canadiens.

\begin{tabular}{|c|c|c|}
\hline $\begin{array}{l}\text { Subscriber group / Groupe } \\
\text { d'abonnés }\end{array}$ & $\begin{array}{l}\text { Print only / Texte imprimé } \\
\text { seulement }\end{array}$ & $\begin{array}{l}\text { Print }+ \text { Online copy } \\
\text { Texte imprimé } \\
\text { et exemplaire électronique }\end{array}$ \\
\hline $\begin{array}{l}\text { Nonmembers within Canada / } \\
\text { Non-membres au Canada }\end{array}$ & $\begin{array}{l}\$ 120.00 \text { per year, plus GST or HST } \\
120,00 \$ \text { par an, plus TPS ou TVH }\end{array}$ & $\begin{array}{l}\$ 160.00 \text { per year, plus GST or HST } \\
160,00 \$ \text { par an, plus TPS ou TVH }\end{array}$ \\
\hline USA / É.-U. & $\begin{array}{l}\$ 150.00 \text { per year } \\
150,00 \$ \text { par an }\end{array}$ & $\begin{array}{l}\$ 190.00 \text { per year } \\
190,00 \$ \text { par an }\end{array}$ \\
\hline Foreign / Étranger & $\begin{array}{l}\$ 190.00 \text { per year } \\
190,00 \$ \text { par an }\end{array}$ & $\begin{array}{l}\$ 230.00 \text { per year } \\
230,00 \$ \text { par an }\end{array}$ \\
\hline
\end{tabular}

More details can be found at www.cjhp-online.ca. If you would like to purchase a subscription, please fill out our CJHP 2014 Subscription Application Form, which can be found on the CJHP website.

Please direct any comments or questions to Colleen Drake, Publications Administrator,

at cdrake@cshp.ca.

Des détails supplémentaires sont fournis à www.cjhp-online.ca. Si vous désirez vous abonner, veuillez remplir le formulaire d'abonnement au JCPH 2014. Vous pouvez l'obtenir en visitant le site Web du JCPH. Pour tout commentaire ou toute question, veuillez vous adresser à Colleen Drake, agente des publications, en écrivant à cdrake@cshp.ca. 